\title{
Persistence and extinction for stochastic delay differential model of prey predator system with hunting cooperation in predators
}

Fathalla A. Rihan ${ }^{1 *}$ and Hebatallah J. Alsakaji ${ }^{1}$

${ }^{*}$ Correspondence:

frihan@uaeu.ac.ae

${ }^{1}$ Department of Mathematical

Sciences, College of Science, UAE

University, Al-Ain, UAE

\section{Springer}

\begin{abstract}
Stochastic differential models provide an additional degree of realism compared to their corresponding deterministic counterparts because of the randomness and stochasticity of real life. In this work, we study the dynamics of a stochastic delay differential model for prey-predator system with hunting cooperation in predators. Existence and uniqueness of global positive solution and stochastically ultimate boundedness are investigated. Some sufficient conditions for persistence and extinction, using Lyapunov functional, are obtained. Illustrative examples and numerical simulations, using Milstein's scheme, are carried out to validate our analytical findings. It is observed that a small scale of white noise can promote the survival of both species; while large noises can lead to extinction of the predator population.
\end{abstract}

Keywords: Extinction; Hunting cooperation; Milstein's numerical scheme; Persistence; Stochastic prey-predator model; Time-delay

\section{Introduction}

Prey-predator (PP) interaction is one of the most extensively studied issues in ecological and mathematical literature; see [1-3]. The classic prey-predator models are mostly variations of the Lotka-Volterra model, which was proposed by Lotka [4] and Volterra [5]. Many studies have explored the effect of predator hunting cooperation on PP systems [68]. Most of these studies utilize deterministic models, which of course supported us with useful results for protecting species. However, the natural growth of populations is always affected by environmental stochastic perturbations which should be taken into account in the process of mathematical modeling. Ecological systems are often subject to environmental noise (e.g., temperature, precipitation), which is an important factor in ecosystems, to suppress a potential population explosion [9]. In reality, natural phenomena counter an environmental noise and usually do not follow deterministic laws strictly but oscillate randomly about some average values, so that the population density never attains a fixed value with the advancement of time $[10,11]$. Therefore, it is useful to see how changes in environment affect the relationship between predator and prey populations. Many authors have investigated this phenomenon (see, e.g., [12-15]).

(c) The Author(s) 2020. This article is licensed under a Creative Commons Attribution 4.0 International License, which permits use sharing, adaptation, distribution and reproduction in any medium or format, as long as you give appropriate credit to the original author(s) and the source, provide a link to the Creative Commons licence, and indicate if changes were made. The images or other third party material in this article are included in the article's Creative Commons licence, unless indicated otherwise in a credit line to the material. If material is not included in the article's Creative Commons licence and your intended use is not permitted by statutory regulation or exceeds the permitted use, you will need to obtain permission directly from the copyright holder. To view a copy of this licence, visit http://creativecommons.org/licenses/by/4.0/. 
A key question in population biology is understanding the conditions under which populations coexist or go extinct. Extinction is one of the most important terms in population dynamics. A species is said to be extinct when the last existing member dies. Therefore, extinction becomes a certainty when there are no surviving individuals that can reproduce and create a new generation. In ecology, extinction is often used informally to refer to local extinction, in which a species ceases to exist in the chosen area of study, but may still exist elsewhere. There are a variety of causes that can contribute directly or indirectly to the extinction of species or group of species, such as lack of food and space or toxic pollution of the entire population habitat, competition for food to better adapted competitors, predation, etc. [16]. Due to the importance of this topic in population dynamics, our main goal in this paper is to investigate persistence and extinction in the considered model.

We should also mention here that time-delays (time-lags) have been extensively introduced into equations used in mathematical ecology to represent the time required for maturation period, reaction time, feeding time, etc. [17-19]. The presence of time-delay in a system greatly affects its stability. It can destabilize the equilibrium points and give rise to a stable limit cycle, oscillations grow, and enrich the dynamics of the model. Incorporating time-delays has been considered by many authors in prey-predator models and biological systems [20-23]. Hutchinson [23] first introduced the delay in a logistic differential equation. He proposed a delay differential model for a single species of the form

$$
\frac{d x(t)}{d t}=r x(t)\left(1-\frac{x(t-\tau)}{K}\right) \quad \text { with } x(\theta)=\phi(\theta)>0, \theta \in[-\tau, 0], \phi(0)>0 .
$$

Here, $r(>0)$ is the intrinsic growth rate and $K(>0)$ is the carrying capacity of the population, and time-delay $\tau$ was considered as hatching time. $\phi(\theta)$ is continuous on $\theta \in[-\tau, 0]$. (This equation is referred to as Hutchinson's equation or delayed logistic equation.)

A simple general two-dimensional delayed model of interaction between prey $x(t)$ and a generalist predator $y(t)$ is represented by

$$
\begin{aligned}
& \frac{d x(t)}{d t}=x(t) \mathcal{G}_{1}\left(x\left(t-\tau_{1}\right), K\right)-y(t) \mathcal{F}(x(t)), \\
& \frac{d y(t)}{d t}=y(t) \mathcal{G}_{2}(y(t))+\mu y(t) \mathcal{F}\left(x\left(t-\tau_{2}\right)\right) .
\end{aligned}
$$

The function $\mathcal{G}_{1}\left(x\left(t-\tau_{1}\right), K\right)$ is logistic per capita growth rate of prey, where $K$ is the environmental carrying capacity, and $\mathcal{G}_{2}(y)$ is the per capita growth rate of predator. $\mathcal{F}(x(t))$ and $\mu \mathcal{F}(x(t))$ are 0 extra responses of predator for a particular prey, and $\mu$ is the conversion efficiency $(0<\mu<1)$. Time-delay $\tau_{1}$ represents the gestation period of the prey or reflects the impact of density-dependent feedback mechanism [24]. Time-delay $\tau_{2}$ is incorporated in the functional response of predator equation to represent the reaction time with the prey. In reality, the reproduction of predators is not immediate to the consumption of prey, as there is some discrete time lag necessary for prey gestation [17].

There exist various and extensive studies of the dynamics of the delayed PP model; see, e.g., [25-28]. In [27], the authors investigated the complex dynamics of a delayed PP system with cooperation among the prey species, they have considered time delays in the growth components for each of the species. Berec [28] assumed a Holling type II functional response of the form $\mathcal{F}(x, y)=\frac{\sigma(y) x}{1+c(y) \sigma(y) x}$, where $\sigma$ is the consumption rate of prey 
Table 1 One biological meaning for the parameters of model (2)

\begin{tabular}{ll}
\hline Parameter & Description \\
\hline$r$ & Intrinsic growth rate \\
$K$ & Environmental carrying capacity \\
$\delta$ & Death rate for predator \\
$\mu$ & Conversion efficiency \\
$\alpha$ & Cooperative hunting parameter \\
$c$ & Handling time of predator \\
$a$ & Predator intra-specific competition rate \\
\hline
\end{tabular}

by their predator and $c$ is the handling time of the predator, both $\sigma$ and $c$ are not constant quantities. Alves et al. [6] considered consumption rate depending on the predator density to implement predator cooperation for searching and capturing the prey. Assuming that $\alpha>0$ is the cooperative 'hunting' parameter, with functional response of the form $\mathcal{F}(x, y)=\frac{(1+\alpha y) x}{1+c(1+\alpha y) x}$, the suggested model takes the form

$$
\begin{aligned}
& \frac{d x(t)}{d t}=r x(t)\left(1-\frac{x\left(t-\tau_{1}\right)}{K}\right)-\frac{[1+\alpha y(t)] x(t) y(t)}{1+c(1+\alpha y(t)) x(t)}, \\
& \frac{d y(t)}{d t}=y(t)(-\delta-a y(t))+\frac{\mu[1+\alpha y(t)] x\left(t-\tau_{2}\right) y(t)}{1+c(1+\alpha y(t)) x\left(t-\tau_{2}\right)},
\end{aligned}
$$

where $\delta>0$ is the death rate of predator and $a>0$ is an intra-specific competition rate for predators. The description of the model parameters is presented in Table 1.

It is known that deterministic models, such as (2), are stable with a cyclic behavior in the common period for the sizes of species populations. However, in practice, stochastic variations will occur in the values of $x$ and $y$, which may produce a qualitatively different behavior. These variations may lead to an extinction of the predator as a result of a possible extinction of the prey. Deterministic models may be inadequate for capturing the exact variability in nature. Then, stochastic models are required for an accurate approximation of the dynamics of such interactions. The random fluctuations result in changing some degree of parameters in the deterministic environment. Many authors have studied stochastic population models and revealed the effects of environmental noises on the dynamics of population models (see [14, 29-31]). Hattaf et al. [14] studied the impact of random noise in the dynamics of delayed SIR epidemic model. They deduced a threshold parameter to determine the extinction and persistence of the disease. However, in [32], the authors studied the effect of environmental fluctuations of a delayed Harrison-type PP model, they analyzed the impact of the combination of delay and noise in the dynamical behavior of the model. In [33], the authors studied the effect of environmental fluctuations on a competitive model for two phytoplankton species where one species liberate toxic substances by considering a discrete time delay parameter in the growth equations of both species.

In this paper, we consider and investigate a stochastic version of PP system (2), so that

$$
\begin{aligned}
& d x(t)=\left[r x(t)\left(1-\frac{x\left(t-\tau_{1}\right)}{K}\right)-\frac{[1+\alpha y(t)] x(t) y(t)}{1+c(1+\alpha y(t)) x(t)}\right] d t+\sigma_{1} x(t) d B_{1}(t), \\
& d y(t)=\left[-\delta y(t)-a y^{2}(t)+\frac{\mu[1+\alpha y(t)] x\left(t-\tau_{2}\right) y(t)}{1+c(1+\alpha y(t)) x\left(t-\tau_{2}\right)}\right] d t+\sigma_{2} y(t) d B_{2}(t) .
\end{aligned}
$$


$B_{1}(t), B_{2}(t)$ are standard independent Wiener processes defined on a complete probability space $\left(\Omega, \mathcal{A},\{\mathcal{A}\}_{t \geq 0}, \mathbf{P}\right)$ with a filtration $\{\mathcal{A}\}_{t \geq 0}$ satisfying the usual conditions; and $\sigma_{1}, \sigma_{2}$ are the positive intensities of white noises.

The rest of this paper is organized as follows. In Sect. 2, we briefly investigate the qualitative behavior for deterministic model (2). In Sect. 3, we study stochastic delay differential equations (SDDEs) (3). Sufficient criteria for global existence, stochastically ultimate boundedness, persistence in mean, and extinction of the system are obtained. Some numerical simulations to validate our mathematical findings are given in Sect. 4, and finally the essential results and their ecological explanations are summarized in Sect. 5.

\section{Deterministic analysis}

Herein, we study the qualitative behavior of the deterministic model. Under some restrictions on the parameters of system (2), there exist three equilibrium points $\mathcal{E}_{0}$, $\mathcal{E}_{1}$, and $\mathcal{E}^{*}$ (see Appendix). We linearize the system around $\mathcal{E}^{*}=\left(x^{*}, y^{*}\right)$, so that $x(t)=x^{*}+\tilde{x}(t)$, $y(t)=y^{*}+\tilde{y}(t)$, then we have

$$
\begin{aligned}
\frac{d \tilde{x}(t)}{d t} & =a_{1} \tilde{x}(t)+a_{2} \tilde{y}+a_{3} \tilde{x}\left(t-\tau_{1}\right), \\
\frac{d \tilde{y}(t)}{d t} & =a_{4} \tilde{y}(t)+a_{5} \tilde{x}\left(t-\tau_{2}\right),
\end{aligned}
$$

where the coefficients are given by

$$
\begin{aligned}
& a_{1}=\frac{c\left(1+\alpha y^{*}\right)^{2} y^{*} x^{*}}{\left(1+c\left(1+\alpha y^{*}\right) x^{*}\right)^{2}}, \quad a_{2}=-\frac{\left(2 \alpha y^{*}+c x^{*}\left(1+\alpha y^{*}\right)^{2}+1\right) x^{*}}{\left(1+c\left(1+\alpha y^{*}\right) x^{*}\right)^{2}}, \quad a_{3}=-\frac{r x^{*}}{K}, \\
& a_{4}=-a y^{*}, \quad a_{5}=\frac{\mu\left(1+\alpha y^{*}\right) y^{*}}{\left(1+c\left(1+\alpha y^{*}\right) x^{*}\right)^{2}} .
\end{aligned}
$$

The characteristic equation of linearization model (4) is given by

$$
\lambda^{2}-\left(a_{1}+a_{4}\right) \lambda+a_{1} a_{4}+\left(a_{3} a_{4}-a_{3} \lambda\right) e^{-\lambda \tau_{1}}-a_{2} a_{5} e^{-\lambda \tau_{2}}=0 .
$$

Let us define a threshold parameter $\mathcal{R}_{0}^{d}=\frac{\mu K}{\delta(1+c K)}$.

Remark 1 The extinction equilibrium $\mathcal{E}_{0}$ is always a saddle point, and the boundary equilibrium point $\mathcal{E}_{1}$ is locally asymptotically stable if $\mathcal{R}_{0}^{d} \equiv \frac{\mu K}{\delta(1+c K)}<1$.

To gain insight regarding interior equilibrium $\mathcal{E}^{*}$, we consider different values of timelags $\tau_{1}$ and $\tau_{2}$ : (i) $\tau_{1}=\tau_{2}=0$, (ii) $\tau_{1}>0, \tau_{2}=0$, (iii) $\tau_{1}=0, \tau_{2}>0$, (iv) $\tau_{1}>0, \tau_{2}>0$.

- Case (i): When $\tau_{1}=\tau_{2}=0$, equation (5) becomes

$$
\lambda^{2}-\left(a_{1}+a_{3}+a_{4}\right) \lambda+a_{1} a_{4}+a_{3} a_{4}-a_{2} a_{5}=0 .
$$

Thus all the roots of (6) have negative real parts if

$$
\left(H_{1}\right) \quad a_{3}+a_{4}<-a_{1} \text { and } a_{1} a_{4}+a_{3} a_{4}>a_{2} a_{5} \text { hold. }
$$


- Case (ii): When $\tau_{2}=0, \tau_{1}>0$, equation (5) becomes

$$
\lambda^{2}-\left(a_{1}+a_{4}\right) \lambda+\left(a_{1} a_{4}-a_{2} a_{5}\right)+\left(a_{3} a_{4}-a_{3} \lambda\right) e^{-\lambda \tau_{1}}=0 .
$$

Let $\lambda=i \omega$ be the root of (7), then it follows that

$$
\begin{aligned}
& -\omega^{2}+\left(a_{1} a_{4}-a_{2} a_{5}\right)=a_{3} \omega \sin \omega \tau_{1}-a_{3} a_{4} \cos \omega \tau_{1}, \\
& -\left(a_{1}+a_{4}\right) \omega=a_{3} \omega \cos \omega \tau_{1}+a_{3} a_{4} \sin \omega \tau_{1}
\end{aligned}
$$

which leads to

$$
\omega^{4}+c_{1} \omega^{2}+c_{2}=0
$$

where $c_{1}=\left(a_{1}+a_{4}\right)^{2}-2\left(a_{1} a_{4}-a_{2} a_{5}\right)-a_{3}^{2}$ and $c_{2}=\left(a_{1} a_{4}-a_{2} a_{5}\right)^{2}-\left(a_{3} a_{4}\right)^{2}$. Thus, equation (9) has at least one positive root $\omega_{1}$ if $c_{2}<0$, therefore we have

$$
\tau_{1, j}=\frac{1}{\omega_{1}}\left\{\arccos \left[\frac{\left(-\omega_{1}^{2}+a_{1} a_{4}-a_{2} a_{5}\right) a_{3} a_{4}+\left(a_{1}+a_{4}\right) a_{3} \omega_{1}^{2}}{a_{3}^{2} \omega_{1}^{2}+\left(a_{3} a_{4}\right)^{2}}\right]+\frac{2 j \pi}{\omega_{1}}\right\}, \quad j=0,1,2, \ldots,
$$

where $j=0,1,2, \ldots$. Thus, $\mathcal{E}^{*}$ remains stable for $\tau_{1}<\tau_{1}^{\prime}$ and unstable for $\tau_{1}>\tau_{1}^{\prime}$ such that $\tau_{1}^{\prime}=\min \left\{\tau_{1, j}\right\}$.

- Case (iii): For $\tau_{1}=0, \tau_{2}>0$, in the same manner, we have

$$
\tau_{2, j}=\frac{1}{\omega_{2}}\left\{\arccos \left[\frac{-\omega_{2}^{2}+a_{1} a_{4}+a_{3} a_{4}}{a_{2} a_{5}}\right]+\frac{2 j \pi}{\omega_{2}}\right\}, \quad j=0,1,2, \ldots,
$$

where $j=0,1,2, \ldots$. Therefore, $\mathcal{E}^{*}$ remains stable for $\tau_{2}<\tau_{2}^{\prime}$ and unstable for $\tau_{2}>\tau_{2}^{\prime}$ such that $\tau_{2}^{\prime}=\min \left\{\tau_{2, j}\right\}$ provided that $\left(a_{1} a_{4}+a_{3} a_{4}\right)^{2}<\left(a_{2} a_{5}\right)^{2}$.

- Case (iv): When $\tau_{1}, \tau_{2}>0$, we assume first that $\tau_{1}$ is varying and $\tau_{2}$ is fixed in its stable interval $\tau_{2} \in\left[0, \tau_{2}^{\prime}\right)$. Assume that there exists a real number $\omega>0$ such that $\lambda=i \omega$ is a root of characteristic equation (5), then separating real and imaginary parts, we get

$$
\begin{aligned}
& -\omega^{2}+a_{1} a_{4}-a_{2} a_{5} \cos \omega \tau_{2}=a_{3} \omega \sin \omega \tau_{1}-a_{3} a_{4} \cos \omega \tau_{1} \\
& -\left(a_{1}+a_{4}\right) \omega+a_{2} a_{5} \sin \omega \tau_{2}=a_{3} \omega \cos \omega \tau_{1}+a_{3} a_{4} \sin \omega \tau_{1}
\end{aligned}
$$

Squaring and adding both sides yields

$$
\omega^{4}+b_{1} \omega^{2}+b_{2} \omega+b_{3}=0
$$

where

$$
\begin{aligned}
& b_{1}=a_{1}^{2}+a_{4}^{2}-a_{3}^{2}+2 a_{2} a_{5} \cos \omega \tau_{2}, \quad b_{2}=-2\left(a_{1}+a_{4}\right) a_{2} a_{5} \sin \omega \tau_{2}, \\
& b_{3}=\left(a_{1} a_{4}\right)^{2}-\left(a_{3} a_{4}\right)^{2}+\left(a_{2} a_{5}\right)^{2}-2 a_{1} a_{2} a_{4} a_{5} \cos \omega \tau_{2} .
\end{aligned}
$$


Equation (13) is a peculiar equation in a complicated form, it is not easy to presume about the nature of the roots. Thus, by applying Descartes rule of signs, we can say that (13) has at least one positive root $\omega_{0}$ if

$$
\left(H_{2}\right) \quad\left(a_{1} a_{4}\right)^{2}+\left(a_{2} a_{5}\right)^{2}<\left(a_{3} a_{4}\right)^{2}+2 a_{1} a_{2} a_{4} a_{5} \cos \omega \tau_{2} .
$$

In this case, we have

$$
\tau_{1, j}=\frac{1}{\omega_{0}}\left\{\arccos \left[\frac{a_{3} \omega_{0}\left(-\left(a_{1}+a_{4}\right) \omega_{0}+a_{2} a_{5} \sin \omega_{0} \tau_{2}\right)-a_{3} a_{4}\left(-\omega_{0}^{2}+a_{1} a_{4}-a_{2} a_{5} \cos \omega_{0} \tau_{2}\right)}{\left(a_{3} \omega_{0}\right)^{2}+\left(a_{3} a_{4}\right)^{2}}\right]+2 j \pi\right\},
$$

where $j=0,1,2, \ldots$ Thus, $\mathcal{E}^{*}$ remains stable for $\tau_{1}<\tau_{1}^{*}$ such that $\tau_{1}^{*}=\min \left\{\tau_{1, j}\right\}$ as in (14).

To check the transversality condition of Hopf bifurcation, we fix $\tau_{2}$ in its stable interval and differentiate equations (12) with respect to $\tau_{1}$. Then, substituting $\tau_{1}=\tau_{1,0}$ and $w=w_{0}$, we have

$$
\begin{aligned}
& \left.\left.\left.A_{2}\left(\frac{d(\Re \lambda)}{d \tau_{1}}\right)\right|_{\tau_{1}=\tau_{1,0}}\right)+\left.A_{1}\left(\frac{d(w)}{d \tau_{1}}\right)\right|_{\tau_{1}=\tau_{1,0}}\right)=A_{3}, \\
& \left.\left.-\left.A_{1}\left(\frac{d(\Re \lambda)}{d \tau_{1}}\right)\right|_{\tau_{1}=\tau_{1,0}}\right)+\left.A_{2}\left(\frac{d(w)}{d \tau_{1}}\right)\right|_{\tau_{1}=\tau_{1,0}}\right)=A_{4},
\end{aligned}
$$

where

$$
\begin{aligned}
& A_{1}=-2 \omega_{0}+\left(-a_{3}-a_{3} a_{4} \tau_{1,0}\right) \sin \omega_{0} \tau_{1,0}+a_{2} a_{5} \tau_{2} \sin \omega_{0} \tau_{2}-a_{3} \tau_{1,0} \omega_{0} \cos \omega_{0} \tau_{1,0}, \\
& A_{2}=\left(a_{1}+a_{4}\right)+\left(a_{3}+a_{3} a_{4} \tau_{1,0}\right) \cos \omega_{0} \tau_{1,0}-a_{3} \omega_{0} \tau_{1,0} \sin \omega_{0} \tau_{1,0}-a_{2} a_{5} \tau_{2} \cos \omega_{0} \tau_{2}, \\
& A_{3}=a_{3} \omega_{0}^{2} \cos \omega_{0} \tau_{1,0}+a_{3} a_{4} \omega_{0} \sin \omega_{0} \tau_{1,0}, \\
& A_{4}=a_{3} a_{4} \omega_{0} \cos \omega_{0} \tau_{1,0}-a_{3} \omega_{0}^{2} \sin \omega_{0} \tau_{1,0} .
\end{aligned}
$$

From (15), we get

$$
\left.\left.\left(\frac{d(\Re \lambda)}{d \tau_{1}}\right)\right|_{\tau_{1}=\tau_{1,0}}\right)=\frac{A_{2} A_{3}-A_{1} A_{4}}{A_{2}^{2}+A_{1}^{2}} .
$$

Assume that

$$
\left(H_{3}\right) \quad A_{2} A_{3}>A_{1} A_{4} \quad \text { holds, }
$$

then a Hopf bifurcation occurs for $\tau_{1}=\tau_{1,0}$.

Therefore, for Case (iv), we arrive at the following theorem.

Theorem 1 Suppose that $\mathcal{E}^{*}$ exists for system (2) and $\left(H_{1}\right)-\left(H_{3}\right)$ hold, such that $\tau_{2} \in\left[0, \tau_{2}^{\prime}\right)$, then there exists a positive threshold parameter $\tau_{1}^{*}$ such that the interior equilibrium $\mathcal{E}^{*}$ is locally asymptotically stable for $\tau_{1}<\tau_{1}^{*}$ and unstable for $\tau_{1}>\tau_{1}^{*}$. Furthermore, system (2) undergoes a Hopf bifurcation at $\mathcal{E}^{*}$ where $\tau_{1}=\tau_{1}^{*}$.

If $\tau_{1}$ is fixed in its stable interval and $\tau_{2}$ varies, we arrive at the following remark. 
Remark 2 If $\tau_{1} \in\left[0, \tau_{1}^{\prime}\right)$, there exists a threshold parameter $\tau_{2}^{*}$ such that the interior equilibrium $\mathcal{E}^{*}$ is locally asymptotically stable for $\tau_{2}<\tau_{2}^{*}$ and unstable for $\tau_{2}>\tau_{2}^{*}$, where $\tau_{2}^{*}=\min \left\{\tau_{2, j}\right\}$ is given by

$$
\tau_{2, j}=\frac{1}{w_{3}} \arccos \left[\frac{a_{3} a_{4} \cos \omega_{3} \tau_{1}-\omega_{3}^{2}-a_{1} a_{4}-a_{3} \omega_{3} \sin \omega_{3} \tau_{1}}{a_{2} a_{5}}\right]+\frac{2 j \pi}{w_{3}}, \quad j=0,1,2, \ldots
$$

\section{Stochastic analysis}

In this section, we extend the analysis to the stochastic model, where we incorporate white noise into the growth equations of both prey and predator. Recall model (3)

$$
\begin{aligned}
& d x(t)=\left[r x(t)\left(1-\frac{x\left(t-\tau_{1}\right)}{K}\right)-\frac{[1+\alpha y(t)] x(t) y(t)}{1+c(1+\alpha y(t)) x(t)}\right] d t+\sigma_{1} x(t) d B_{1}(t), \\
& d y(t)=\left[-\delta y(t)-a y^{2}(t)+\frac{\mu[1+\alpha y(t)] x\left(t-\tau_{2}\right) y(t)}{1+c(1+\alpha y(t)) x\left(t-\tau_{2}\right)}\right] d t+\sigma_{2} y(t) d B_{2}(t) .
\end{aligned}
$$

We assume that $\kappa \in[-\tau, 0], \tau=\max \left\{\tau_{1}, \tau_{2}\right\}$, i.e., $\left(x_{0}, y_{0}\right)=\left(\phi_{1}, \phi_{2}\right)^{T} \in C\left([-\tau, 0], \mathbb{R}_{+}^{2}\right)$ with $\mathbb{R}_{+}^{2}=\left\{(x, y) \in \mathbb{R}^{2}: x>0, y>0\right\}$, if $(x, y) \in \mathbb{R}^{2}$, its norm is denoted by $|(x, y)|=\sqrt{x^{2}+y^{2}}$. The initial value of system (17) becomes

$$
(x(\kappa), y(\kappa))=\{(x(\kappa), y(\kappa)):-\tau \leq \kappa \leq 0\} \in C\left([-\tau, 0] ; \mathbb{R}_{+}^{2}\right) .
$$

Now, we investigate the existence and uniqueness of positive solutions.

\subsection{Existence and uniqueness of positive solution}

In order to prove that the model of SDDEs (17) has a unique global solution (i.e., no explosion in a finite-time) for any given initial condition, the coefficients of system (17) are generally required to satisfy the linear growth condition and local Lipschitz condition [34, 35].

To show that model (17) has a global positive solution, let us firstly prove that the model has a positive local solution by making the change of variables. Then we prove that this solution will also not explode to infinity at any finite time by using a suitable stochastic Lyapunov functional.

Theorem 2 Let the coefficients of system (17) be locally Lipschitz continuous, then for any given initial data (18) there is a unique positive solution $(x(t), y(t))$ of system (17) on $t \geq-\tau$, and the solution will remain in $\mathbb{R}_{+}^{2}$ with probability one.

Proof Let $n(t)=\ln x(t), p(t)=\ln y(t)$, we have the system

$$
\begin{aligned}
& d n(t)=\left(r-\frac{r}{K} e^{n\left(t-\tau_{1}\right)}-\frac{\left(1+\alpha e^{p(t)}\right) e^{n(t)}}{1+c\left(1+\alpha e^{p(t)}\right) e^{n(t)}}-\frac{\sigma_{1}^{2}}{2}\right) d t+\sigma_{1} d B_{1}(t), \\
& d p(t)=\left(\frac{\mu\left(1+\alpha e^{p(t)}\right) e^{n\left(t-\tau_{2}\right)}}{1+c\left(1+\alpha e^{p(t)}\right) e^{n\left(t-\tau_{2}\right)}}-\delta-a e^{p(t)}-\frac{\sigma_{2}^{2}}{2}\right) d t+\sigma_{2} d B_{2}(t),
\end{aligned}
$$

for any initial values $n(\kappa)=\ln x(\kappa), p(\kappa)=\ln y(\kappa), \kappa \in[-\tau, 0]$. It is easy to show that all the coefficients of (19) satisfy the local Lipschitz condition; therefore, there is a unique local 
solution $(n(t), p(t))$ on $\left[-\tau, \tau_{e}\right)$, where $\tau_{e}$ is explosion time [36]. By Ito's formula, we can see that $x(t)=e^{n(t)}, y(t)=e^{p(t)}$; therefore, there is a unique local positive solution of (17) for any given initial value $\left(x_{0}, y_{0}\right) \in \mathbb{R}_{+}^{2}$.

To show that this solution is global, we need to show $\tau_{e}=\infty$ a.s. (almost surely). Let $l_{0}>0$ be sufficiently large so that $(x(t), y(t))=\left\{\left(\phi_{1}(t), \phi_{2}(t)\right):-\tau \leq t \leq 0\right\} \in C\left([-\tau, 0] ; \mathbb{R}_{+}^{2}\right)$ all lie within the interval $\left[\frac{1}{l_{0}}, l_{0}\right]$. Now, for each integer $l \geq l_{0}$, define the stopping time $\tau_{l}=\inf \left\{t \in\left[-\tau, \tau_{e}\right): x(t) \notin\left(\frac{1}{l}, l\right), y(t) \notin\left(\frac{1}{l}, l\right)\right\}$, let inf $\phi=\infty$. $\tau_{l}$ is increasing with $l$ and let $\tau_{\infty}=\lim _{l \rightarrow \infty} \tau_{l}$, then $\tau_{\infty} \leq \tau_{e}$. By showing $\tau_{\infty}=\infty$ a.s., we want to conclude that $\tau_{e}=\infty$ a.s. If this assertion is erroneous, then there exists a pair of constants $T>0$ and $\epsilon \in(0,1)$ such that $P\left\{\tau_{\infty} \leq T\right\}>\epsilon$. Therefore, there is an integer $l_{1} \geq l_{0}$ such that

$$
P\left\{\tau_{l} \leq T\right\}>\epsilon \quad \text { for all } l \geq l_{1} .
$$

Define a $\mathcal{C}^{2}$-function $V(x, y): \mathbb{R}_{+} \times \mathbb{R}_{+} \rightarrow \mathbb{R}_{+}$by

$$
V(x, y)=(x-\log x-1)+(y-\log y-1)+\frac{r}{K} \int_{t}^{t+\tau_{1}} x\left(s-\tau_{1}\right) d s .
$$

Clearly, this function is nonnegative for all $x, y \geq 0$. Let $l \geq l_{0}$ and $T>0$ be arbitrary. For $0 \leq t \leq \tau_{l} \wedge T$, by Itô's formula for $V$, we get

$$
\begin{aligned}
d V= & {\left[(x-1)\left[r-\frac{r}{K} x\left(t-\tau_{1}\right)-\frac{(1+\alpha y) y}{1+c(1+\alpha y) x}\right]\right.} \\
& +(y-1)\left[\frac{\mu(1+\alpha y) x\left(t-\tau_{2}\right)}{1+c(1+\alpha y) x\left(t-\tau_{2}\right)}-\delta-a y\right]+\frac{\sigma_{1}^{2}+\sigma_{2}^{2}}{2} \\
& \left.+\frac{r}{K} x-\frac{r}{K} x\left(t-\tau_{1}\right)\right] d t+\sigma_{1}(x-1) d B_{1}(t)+\sigma_{2}(y-1) d B_{2}(t) \\
\leq & {\left[-a y^{2}+(1+\mu+a-\delta) y+r \frac{K+1}{K} x+(\delta-r)+\frac{\sigma_{1}^{2}+\sigma_{2}^{2}}{2}\right] d t } \\
& +\sigma_{1}(x-1) d B_{1}(t)+\sigma_{2}(y-1) d B_{2}(t) \\
\leq & \gamma d t+\sigma_{1}(x-1) d B_{1}(t)+\sigma_{2}(y-1) d B_{2}(t),
\end{aligned}
$$

where $\gamma$ is a positive number. Therefore

$$
\int_{\tau_{l} \wedge T-\tau}^{\tau_{l} \wedge T} d V(x, y) \leq \int_{\tau_{l} \wedge T-\tau}^{\tau_{l} \wedge T} \gamma d t+\int_{\tau_{l} \wedge T-\tau}^{\tau_{l} \wedge T} \sigma_{1}(x-1) d B_{1}(t)+\int_{\tau_{l} \wedge T-\tau}^{\tau_{l} \wedge T} \sigma_{2}(y-1) d B_{2}(t) .
$$

Taking expectation of both sides implies

$$
\mathbf{E}\left[V\left(x\left(t_{l} \wedge T\right), y\left(t_{l} \wedge T\right)\right)\right] \leq V(x(0), y(0))+\gamma T .
$$

Set $\Omega_{l}=\left\{\tau_{l} \leq T\right\}$ for $l \geq l_{1}$ and by the virtue of (20), we obtain $\mathcal{P}\left(\Omega_{l}\right) \geq \epsilon$. For every $\eta \in \Omega_{l}$, $x\left(\tau_{l}, \eta\right)$ and $y\left(\tau_{l}, \eta\right)$ equal either $l$ or $\frac{1}{l}$, Consequently, $V\left(x\left(\tau_{l}, \eta\right), y\left(\tau_{l}, \eta\right)\right)$ is no less than either $l-\log l-1$ or $\frac{1}{l}+\log l-1$. Therefore, we can get

$$
V\left(x\left(\tau_{l}, \eta\right), y\left(\tau_{l}, \eta\right)\right) \geq \min \left\{l-\log l-1, \frac{1}{l}+\log l-1\right\} .
$$


It follows from (22) that

$$
\begin{aligned}
V(x(0), y(0))+\gamma T & \geq \mathbf{E}\left[1_{\Omega_{l}}(\eta) V\left(x\left(\tau_{l}\right), y\left(\tau_{l}\right)\right)\right] \\
& \geq \epsilon[l-\log l-1] \wedge\left[\frac{1}{l}+\log l-1\right],
\end{aligned}
$$

where $1_{\Omega_{l}}$ is the indicator function of $\Omega_{l}$. Letting $l \rightarrow \infty$ leads to a contradiction that $\infty>V(x(0), y(0))+\gamma T=\infty$. Therefore, we get $\tau_{\infty}=\infty$ a.s.

\subsection{Stochastically ultimate boundedness}

After discussion on the existence and uniqueness of positive solution of SDDEs (17), we show that the positive solution does not explode to infinity in a finite time.

Definition 1 ([37]) The solution $(x(t), y(t))$ of SDDEs (17) is said to be stochastically ultimately bounded a.s. if, for any $\epsilon \in(0,1)$, there is a positive constant $\varphi=\varphi(\epsilon)$ such that $\lim _{t \rightarrow \infty} \sup \mathbf{P}\{|(x(t), y(t))|>\varphi\}<\epsilon$.

Theorem 3 If any $\theta \in(0,1)$ and $\mu \theta\left(\mu K e^{\tau_{2}}-\delta\right)>a$, and there exists a positive constant $N=N(\theta)$, which is independent of the initial value (18), such that

$$
\lim _{t \rightarrow \infty} \sup \mathbf{E}|(x(t), y(t))|^{\theta} \leq N
$$

then the positive solution $(x(t), y(t))$ of system (17) is stochastically ultimately bounded.

Proof To prove (23), define

$$
V(x, y)=x^{\theta}+y^{\theta}, \quad(x, y) \in \mathbb{R}_{+}^{2} .
$$

Applying Itô's formula gives

$$
\begin{aligned}
L V(x, y)= & \theta x^{\theta}\left[r-\frac{r}{K} x\left(t-\tau_{1}\right)-\frac{(1+\alpha y) y}{1+c(1+\alpha y) x}\right]+\frac{\sigma_{1}^{2}}{2} \theta(\theta-1) x^{\theta} \\
& +\theta y^{\theta}\left[\frac{\mu(1+\alpha y) x\left(t-\tau_{2}\right)}{1+c(1+\alpha y) x\left(t-\tau_{2}\right)}-\delta-a y\right]+\frac{\sigma_{2}^{2}}{2} \theta(\theta-1) y^{\theta} .
\end{aligned}
$$

Denote

$$
\begin{aligned}
L V(x, y) \leq & \theta x^{\theta}\left(r-\frac{r}{K} x\left(t-\tau_{1}\right)\right)-\frac{\sigma_{1}^{2}}{2} \theta(1-\theta) x^{\theta}+\frac{\mu \theta y^{\theta}(1+\alpha y) x\left(t-\tau_{2}\right)}{1+c(1+\alpha y) x\left(t-\tau_{2}\right)} \\
& -\frac{\sigma_{2}^{2}}{2} \theta(1-\theta) y^{\theta}-\theta a y^{\theta+1} \\
\leq & r \theta x^{\theta}-\frac{\sigma_{1}^{2}}{2} \theta(1-\theta) x^{\theta}-\frac{\sigma_{2}^{2}}{2} \theta(1-\theta) y^{\theta}+\mu \theta\left(\frac{\mu K e^{\tau_{2}}-\delta}{a}\right)\left|x\left(t-\tau_{2}\right)\right|^{2} \\
= & H(x, y)-V(x, y)-e^{\tau_{2}}|x(t)|^{2}+\mu \theta\left(\frac{\mu K e^{\tau_{2}}-\delta}{a}\right)\left|x\left(t-\tau_{2}\right)\right|^{2}
\end{aligned}
$$

where

$$
H(x, y)=(r \theta+1) x^{\theta}+y^{\theta}-\frac{\sigma_{1}^{2}}{2}(1-\theta) x^{\theta}-\frac{\sigma_{2}^{2}}{2} \theta(1-\theta) y^{\theta}+e^{\tau_{2}}|x(t)|^{2} \leq N_{0}
$$


for $(x, y) \in \mathbb{R}_{+}^{2}$. Note that $H(x, y)$ is bounded in $\mathbb{R}_{+}^{2}$. Hence, we have

$$
L V(x, y) \leq N_{0}-V(x, y)-e^{\tau_{2}}|x(t)|^{2}+\frac{\mu \theta\left(\mu K e^{\tau_{2}}-\delta\right)}{a}\left|x\left(t-\tau_{2}\right)\right|^{2}
$$

Thus, we obtain

$$
\begin{aligned}
d V(x, y)= & L V(x, y) d t+\sigma_{1} \theta x^{\theta} d B_{1}(t)+\sigma_{2} \theta y^{\theta} d B_{2}(t) \\
\leq & \left(N_{0}-V(x, y)-e^{\tau_{2}}|x(t)|^{2}+\frac{\mu \theta\left(\mu K e^{\tau_{2}}-\delta\right)}{a}\left|x\left(t-\tau_{2}\right)\right|^{2}\right) d t \\
& +\sigma_{1} \theta x^{\theta} d B_{1}(t)+\sigma_{2} \theta y^{\theta} d B_{2}(t) .
\end{aligned}
$$

Again, using Itô's formula, we have

$$
\begin{aligned}
d\left(e^{t} V(x, y)\right)= & e^{t} V(x, y) d t+e^{t} d V(x, y) \\
\leq & e^{t}\left[N_{0}-e^{\tau_{2}}|x(t)|^{2}+\frac{\mu \theta\left(\mu K e^{\tau_{2}}-\delta\right)}{a}\left|x\left(t-\tau_{2}\right)\right|^{2}\right] d t \\
& +e^{t} \sigma_{1} \theta x^{\theta} d B_{1}(t)+e^{t} \sigma_{2} \theta y^{\theta} d B_{2}(t) .
\end{aligned}
$$

If $\mu \theta\left(\mu K e^{\tau_{2}}-\delta\right)>a$, then

$$
\begin{aligned}
e^{t} \mathbf{E} V(x, y) \leq & V(x(0), y(0))+N_{0} e^{t} \\
& -\mathbf{E} \int_{0}^{t} e^{s+\tau_{2}}|x(s)|^{2} d s+\mu \theta \frac{\mu K e^{\tau_{2}}-\delta}{a} \mathbf{E} \int_{0}^{t} e^{s}\left|x\left(s-\tau_{2}\right)\right|^{2} d s \\
= & V(x(0), y(0))+N_{0} e^{t}-\mathbf{E} \int_{0}^{t} e^{s+\tau_{2}}|x(s)|^{2} d s+\frac{\mu^{2} \theta K}{a} \mathbf{E} \int_{-\tau_{2}}^{t-\tau_{2}} e^{s+\tau_{2}}|x(s)|^{2} d s \\
& -\frac{\delta \mu \theta}{a} \mathbf{E} \int_{0}^{t} e^{s}\left|x\left(s-\tau_{2}\right)\right|^{2} d s \\
\leq & V(x(0), y(0))+N_{0} e^{t}+\frac{\mu^{2} \theta K}{a} \mathbf{E} \int_{-\tau_{2}}^{0} e^{s+\tau_{2}}|x(s)|^{2} d s,
\end{aligned}
$$

which implies that

$$
\lim _{t \rightarrow \infty} \sup \mathbf{E} V(x(t), y(t)) \leq N_{0} .
$$

We get

$$
\lim _{t \rightarrow \infty} \sup \mathbf{E}|x(t), y(t)|^{\theta} \leq \sqrt{2^{\theta}} \lim _{t \rightarrow \infty} \sup \mathbf{E} V(x(t), y(t)) \leq \sqrt{2^{\theta}} N_{0}=N(\theta) .
$$

Since $\lim _{t \rightarrow \infty} \sup \mathbf{E}|x(t), y(t)|^{\theta} \leq N$, then, for any $\epsilon>0$, let $\varphi=N^{2} / \epsilon^{2}$. By Chebyshev's inequality

$$
\mathbf{P}\{|(x(t), y(t))|>\varphi\} \leq \frac{\mathbf{E}(\sqrt{|(x(t), y(t))|})}{\sqrt{\varphi}},
$$


we obtain

$$
\lim _{t \rightarrow \infty} \sup \mathbf{P}\{|(x(t), y(t))|>\varphi\} \leq \frac{N}{\sqrt{\varphi}}:=\epsilon .
$$

Thus, model (17) is stochastically ultimately bounded.

\subsection{Persistence}

Under certain restrictions on the parameter values with small intensities of white noise, we investigate the conditions under which the persistence of system SDDEs (17) occurs.

Let us first define persistence in the mean of a dynamical system.

Definition 2 The species $y(t)$ is said to be persistent (see [16]) in the mean if

$$
\lim _{t \rightarrow \infty} \frac{1}{t} \int_{0}^{t} y(s) d s>0 \quad \text { a.s. }
$$

In order to show the persistence, we go through some necessary lemmas.

Lemma 1 ([38]) Let $M=\left\{M_{t}\right\}_{t \geq 0}$ be a real-valued continuous local martingale vanishing at $t=0$. Then

$$
\lim _{t \rightarrow \infty}\langle M, M\rangle_{t}=\infty \quad \text { a.s. } \quad \Rightarrow \quad \lim _{t \rightarrow \infty} \frac{M_{t}}{\langle M, M\rangle_{t}}=0 \quad \text { a.s. }
$$

and also

$$
\lim \sup _{t \rightarrow \infty} \frac{\langle M, M\rangle_{t}}{t}<\infty \quad \text { a.s. } \Rightarrow \lim _{t \rightarrow \infty} \frac{M_{t}}{t}=0 \quad \text { a.s., }
$$

where $\langle M, M\rangle_{t}$ denotes the quadratic variation of $M$.

Lemma $2([39])$ Let $y(t) \in \mathbb{C}[[0, \infty) \times \Omega,(0, \infty)]$. If there exist positive constants $\lambda_{0}, \lambda$ such that

$$
\ln y(t) \geq \lambda t-\lambda_{0} \int_{0}^{t} y(s) d s+F(t) \quad \text { a.s. }
$$

for all $t \geq 0$, where $F \in \mathbb{C}[[0, \infty) \times \Omega, \mathbb{R}]$ and $\lim _{t \rightarrow \infty} \frac{F(t)}{t}=0$ a.s., then

$$
\lim _{t \rightarrow \infty} \frac{1}{t} \int_{0}^{t} y(s) d s \geq \frac{\lambda}{\lambda_{0}} \quad \text { a.s. }
$$

Let us define a threshold parameter $\mathcal{R}_{0}^{s}$ as follows:

$$
\mathcal{R}_{0}^{s}=\frac{\mu K}{\tilde{\delta}(1+c)}>0, \quad \text { where } \tilde{\delta}=\delta+\frac{\sigma_{2}^{2}}{2} .
$$

Theorem 4 Let $(x(t), y(t))$ be the solution of SDDEs (17) with initial conditions (18). Assume that $2 r>\sigma_{1}^{2}$, then system (17) will be persistent if $\mathcal{R}_{0}^{s}>1$, so that $\liminf _{t \rightarrow \infty} \frac{1}{t} \times$ $\int_{0}^{t} y(s) d s>0$, a.s. 
Proof Using Itô's formula to the first equation of system (17) yields

$$
d\left(\ln x(t)-\frac{r}{K} \int_{t}^{t+\tau_{1}} x\left(s-\tau_{1}\right) d s\right) \leq\left(\left(r-\frac{\sigma_{1}^{2}}{2}\right)-\frac{r}{K} x(t)\right) d t+\sigma_{1} d B_{1}(t)
$$

Integrating inequality (27) from 0 to $t$ results in

$$
\begin{aligned}
& \frac{\ln x(t)-\frac{r}{K} \int_{t}^{t+\tau_{1}} x\left(s-\tau_{1}\right) d s}{t}-\frac{\ln x(0)-\frac{r}{K} \int_{0}^{\tau_{1}} x\left(s-\tau_{1}\right) d s}{t} \\
& \quad \leq\left(r-\frac{\sigma_{1}^{2}}{2}\right)-\frac{r}{K}\langle x(t)\rangle+\frac{\sigma_{1} B_{1}(t)}{t} .
\end{aligned}
$$

Thus,

$$
\langle x(t)\rangle \leq \frac{K}{r}\left(r-\frac{\sigma_{1}^{2}}{2}\right)+\gamma_{1}(t)
$$

where

$$
\gamma_{1}(t)=\frac{K}{r}\left[\frac{\sigma_{1} B_{1}(t)}{t}-\frac{\ln x(t)-\frac{r}{K} \int_{t}^{t+\tau_{1}} x\left(s-\tau_{1}\right) d s}{t}+\frac{\ln x(0)-\frac{r}{K} \int_{0}^{\tau_{1}} x\left(s-\tau_{1}\right) d s}{t}\right],
$$

it follows from Lemma 1 that

$$
\lim _{t \rightarrow \infty} \frac{B_{1}(t)}{t}=0 \quad \text { a.s. }
$$

Note

$$
\frac{\int_{t}^{t+\tau_{1}} x\left(s-\tau_{1}\right) d s}{t}=\frac{1}{t} \int_{t-\tau_{1}}^{t} x(s) d s=\frac{1}{t}\left[\int_{0}^{t} x(s) d s-\int_{0}^{t-\tau_{1}} x(s) d s\right] .
$$

Therefore, $\lim _{t \rightarrow \infty} \int_{t}^{t+\tau_{1}} \frac{x\left(s-\tau_{1}\right) d s}{t}=0$. Moreover, $\lim _{t \rightarrow \infty} \int_{0}^{\tau_{1}} \frac{x\left(s-\tau_{1}\right) d s}{t}=\lim _{t \rightarrow \infty} \frac{\int_{-\tau_{1}}^{0} \phi_{1}(t) d t}{t}=$ 0 . Thus, we obtain

$$
\lim _{t \rightarrow \infty} \gamma_{1}=0 \quad \text { a.s. }
$$

By Itô's formula, we get

$$
\begin{aligned}
& d\left(\ln x(t)-\frac{r}{K} \int_{t}^{t+\tau_{1}} x\left(s-\tau_{1}\right) d s\right) \\
& \quad=\left[r\left(1-\frac{x\left(t-\tau_{1}\right)}{K}\right)-\frac{(1+\alpha y) y(t)}{1+c(1+\alpha y) x(t)}-\frac{r}{K} x(t)+\frac{r}{K} x\left(t-\tau_{1}\right)-\frac{\sigma_{1}^{2}}{2}\right] d t+\sigma_{1} d B_{1}(t) \\
& \quad \geq\left[r-\frac{r}{K} x(t)-2(1+\alpha) y(t)-\frac{\sigma_{1}^{2}}{2}\right] d t+\sigma_{1} d B_{1}(t)
\end{aligned}
$$


so we have

$$
\begin{gathered}
\frac{\ln x(t)-\frac{r}{K} \int_{t}^{t+\tau_{1}} x\left(s-\tau_{1}\right) d s}{t}-\frac{\ln x(0)-\frac{r}{K} \int_{0}^{\tau_{1}} x\left(s-\tau_{1}\right) d s}{t} \\
\geq\left(r-\frac{\sigma_{1}^{2}}{2}\right)-2(1+\alpha)\langle y\rangle-\frac{r}{K}\langle x\rangle+\frac{\sigma_{1} B_{1}(t)}{t} .
\end{gathered}
$$

Therefore,

$$
\langle x(t)\rangle \geq-\frac{2 K(1+\alpha)}{r}\langle y(t)\rangle+\frac{K}{r}\left(r-\frac{\sigma_{1}^{2}}{2}\right)+\gamma_{1}(t) .
$$

Let

$$
V=\ln y(t)+\int_{t}^{t+\tau_{2}}\left[\frac{\mu x\left(s-\tau_{2}\right)}{1+c x\left(s-\tau_{2}\right)}\right] d s
$$

utilizing Itô's formula, we obtain

$$
d V=\left[\frac{\mu(1+\alpha y) x\left(t-\tau_{2}\right)}{1+c(1+\alpha y) x\left(t-\tau_{2}\right)}-\delta-a y-\frac{1}{2} \sigma_{2}^{2}+\frac{\mu x(t)}{1+c x(t)}-\frac{\mu x\left(t-\tau_{2}\right)}{1+c x\left(t-\tau_{2}\right)}\right] d t+\sigma_{2} d B_{2}(t) .
$$

According to (32), we have

$$
d V \geq\left[\frac{\mu x(t)}{1+c x(t)}-\delta-a y-\frac{1}{2} \sigma_{2}^{2}\right] d t+\sigma_{2} d B_{2}(t)
$$

- Case (1): When $x(t) \leq 1$, then

$$
\begin{aligned}
& d V \geq\left[\frac{\mu x(t)}{1+c}-\delta-a y-\frac{1}{2} \sigma_{2}^{2}\right] d t+\sigma_{2} d B_{2}(t) . \\
& \frac{V(t)-V(0)}{t} \geq \frac{\mu}{1+c}\langle x(t)\rangle-a\langle y(t)\rangle-\left[\delta+\frac{1}{2} \sigma_{2}^{2}\right]+\frac{\sigma_{2}}{t} B_{2}(t) .
\end{aligned}
$$

Substituting (30) into (35), we obtain

$$
\frac{V(t)-V(0)}{t} \geq \frac{\mu}{1+c}\left[\frac{-2 K(1+\alpha)}{r}\langle y(t)\rangle+K\left(1-\frac{\sigma_{1}^{2}}{2 r}\right)+\gamma_{1}(t)\right]-a\langle y(t)\rangle-\tilde{\delta}+\frac{\sigma_{2}}{t} B_{2}(t) .
$$

Therefore,

$$
\begin{aligned}
\ln y(t) \geq & {\left[\frac{\mu K}{(1+c)}\left(1-\frac{\sigma_{1}^{2}}{2 r}\right)-\tilde{\delta}\right] t-\left[\frac{2 \mu K(1+\alpha)}{r(1+c)}+a\right]\langle y(t)| t+\frac{\mu}{1+c} \gamma_{1}(t) t+\sigma_{2} B_{2}(t) } \\
& +\ln y(0)+\int_{0}^{\tau_{2}} \frac{\mu x\left(s-\tau_{2}\right)}{1+c x\left(s-\tau_{2}\right)} d s-\int_{t}^{t+\tau_{2}} \frac{\mu x\left(t-\tau_{2}\right)}{1+c x\left(t-\tau_{2}\right)} d s \\
= & : \lambda t-\lambda_{0} \int_{0}^{t} y(s) d s+F(t),
\end{aligned}
$$


where

$$
\begin{aligned}
& \lambda=\frac{\mu K}{(1+c)}\left(1-\frac{\sigma_{1}^{2}}{2 r}\right)-\tilde{\delta}, \quad \lambda_{0}=\frac{2 \mu K(1+\alpha)}{r(1+c)}+a \\
& F(t)=\frac{\mu}{1+c} \gamma_{1}(t) t+\sigma_{2} B_{2}(t)+\ln y(0)+\int_{0}^{\tau_{2}} \frac{\mu x\left(s-\tau_{2}\right)}{1+c x\left(s-\tau_{2}\right)} d s-\int_{t}^{t+\tau_{2}} \frac{\mu x\left(t-\tau_{2}\right)}{1+c x\left(t-\tau_{2}\right)} d s .
\end{aligned}
$$

Since $2 r>\sigma_{1}^{2}$ and $\mathcal{R}_{0}^{s}>1$, this implies that $\lambda>0$. Using this together with (29) and Lemma 2, one obtains

$$
\lim \inf _{t \rightarrow \infty}\langle y(t)\rangle \geq \frac{r(1+c)}{2 \mu K(1+\alpha)+\operatorname{ar}(1+c)}\left(\frac{\mu K\left(2 r-\sigma_{1}^{2}\right)}{2 r(1+c)}-\tilde{\delta}\right)>0
$$

- Case (2): When $x(t)>1$, from (33) we can get

$$
\begin{aligned}
& d V \geq\left[\frac{\mu}{1+c}-\delta-a y-\frac{1}{2} \sigma_{2}^{2}\right] d t+\sigma_{2} d B_{2}(t), \\
& \frac{V(t)-V(0)}{t} \geq \frac{\mu}{1+c}-a\langle y(t)\rangle-\tilde{\delta}+\frac{\sigma_{2}}{t} B_{2}(t) .
\end{aligned}
$$

Since $\mathcal{R}_{0}^{s}>1$, and following a similar proof to Case (1), we can obtain

$$
\lim _{t \rightarrow \infty}\langle y(t)\rangle \geq \frac{1}{a}\left(\frac{\mu}{1+c}-\tilde{\delta}\right)>0
$$

This completes the proof.

\subsection{Extinction}

Extinction is one of the most important terms in population dynamics. A species is said to be extinct if there is no existing member in the habitat. Although, under some conditions, the solution to the original deterministic DDEs (2) may be persistent, the solution to the associated SDDEs will become extinct with probability one. This reveals the important fact that the environmental noise may make the population extinct. Now, we establish the conditions under which extinction of predator population occurs.

Definition 3 The species $y(t)$ is said to go to extinction with probability one if $\lim _{t \rightarrow \infty} y(t)=0$ a.s.

Theorem 5 Let $a>\mu \alpha$. If $\mathcal{R}_{0}^{s}<1$, then the solution $(x(t), y(t))$ of model (17), for any given initial value (18), satisfies

$$
\lim \sup _{t \rightarrow \infty} \frac{\ln y(t)}{t}<0 \quad \text { a.s. }
$$

which means $\lim _{t \rightarrow \infty} y(t)=0$ exponentially almost surely. In other words, the predators die out with probability one. In addition,

$$
\lim _{t \rightarrow \infty}\langle x(t)\rangle=K\left(1-\frac{\sigma_{1}^{2}}{2 r}\right)
$$


Proof According to (32), we have

$$
\begin{aligned}
d V & \leq\left[\frac{\mu(1+\alpha y) x\left(t-\tau_{2}\right)}{1+c x\left(t-\tau_{2}\right)}-\delta-a y-\frac{1}{2} \sigma_{2}^{2}+\frac{\mu x(t)}{1+c x(t)}-\frac{\mu x\left(t-\tau_{2}\right)}{1+c x\left(t-\tau_{2}\right)}\right] d t+\sigma_{2} d B_{2}(t) \\
& \leq\left[\frac{\mu x(t)}{1+c x(t)}-\delta-(a-\mu \alpha) y(t)-\frac{1}{2} \sigma_{2}^{2}\right] d t+\sigma_{2} d B_{2}(t)
\end{aligned}
$$

since $(a>\mu \alpha)$, then

$$
d V \leq\left[\frac{\mu x(t)}{1+c x(t)}-\delta-\frac{1}{2} \sigma_{2}^{2}\right] d t+\sigma_{2} d B_{2}(t)
$$

Thus, we have two cases.

- When $x(t) \leq 1$, according to (42), we can get

$$
d V \leq\left[\frac{\mu}{1+c}-\tilde{\delta}\right] d t+\sigma_{2} d B_{2}(t)
$$

therefore, we have

$$
\frac{V(t)-V(0)}{t} \leq \frac{\mu}{1+c}-\tilde{\delta}+\frac{\sigma_{2}}{t} B_{2}(t)
$$

So,

$$
\frac{\ln y(t)}{t} \leq \frac{\mu}{1+c}-\tilde{\delta}+\chi_{1}(t)
$$

where

$$
\chi_{1}(t)=\frac{\sigma_{2}}{t} B_{2}(t)+\frac{\ln y(0)}{t}+\frac{1}{t} \int_{0}^{\tau_{2}} \frac{\mu x\left(s-\tau_{2}\right)}{1+c x\left(t-\tau_{2}\right)} d s-\frac{1}{t} \int_{t}^{t+\tau_{2}} \frac{\mu x\left(s-\tau_{2}\right)}{1+c x\left(s-\tau_{2}\right)} d s
$$

In view of the strong law of large numbers of Brownian motion, we can easily obtain that

$$
\lim _{t \rightarrow \infty} \chi_{1}(t)=0 \quad \text { a.s. }
$$

Thus, it follows from (45) and since $\mathcal{R}_{0}^{s}<1$

$$
\lim \sup _{t \rightarrow \infty} \frac{\ln y(t)}{t} \leq \frac{\mu}{1+c}-\tilde{\delta}<0 \quad \text { a.s. }
$$

- When $x(t)>1$, by (42), we have

$$
d V \leq\left[\frac{\mu x(t)}{1+c}-\tilde{\delta}\right] d t+\sigma_{2} d B_{2}(t)
$$

then

$$
\frac{V(t)-V(0)}{t} \leq \frac{\mu K}{1+c}-\frac{\mu}{1+c} \gamma_{1}(t)-\tilde{\delta}+\frac{\sigma_{2}}{t} B_{2}(t)
$$


Therefore,

$$
\frac{\ln y(t)}{t} \leq \frac{\mu K}{1+c}-\tilde{\delta}+\chi_{2}(t)
$$

where

$$
\begin{aligned}
\chi_{2}(t)= & \frac{\sigma_{2}}{t} B_{2}(t)+\frac{\ln y(0)}{t}+\frac{1}{t} \int_{0}^{\tau_{2}} \frac{\mu x\left(s-\tau_{2}\right)}{1+c x\left(s-\tau_{2}\right)} d s-\frac{1}{t} \int_{t}^{t+\tau_{2}}\left(\frac{\mu x\left(s-\tau_{2}\right)}{1+c x\left(s-\tau_{2}\right)} d s\right. \\
& -\frac{\mu}{1+c} \gamma_{1}(t) .
\end{aligned}
$$

In view of the strong law of large numbers of Brownian motion, we can easily obtain that

$$
\lim _{t \rightarrow \infty} \chi_{2}(t)=0 \quad \text { a.s. }
$$

Therefore,

$$
\lim \sup _{t \rightarrow \infty} \frac{\ln y(t)}{t} \leq \frac{\mu K}{1+c}-\tilde{\delta}<0 \quad \text { a.s. }
$$

which implies that $y(t)$ tends to zero exponentially with probability one,

$$
\lim _{t \rightarrow \infty} y(t)=0 \quad \text { a.s. }
$$

By taking the limit on both sides of (28) and (30) at the same time, we can get

$$
\lim _{t \rightarrow \infty}\langle x(t)\rangle=K\left(1-\frac{\sigma_{1}^{2}}{2 r}\right) .
$$

This completes the proof.

\section{Numerical simulations}

In this section, we attempt to validate the mathematical results obtained in the previous sections. We utilize Milstein's scheme with a strong order of convergence one discussed in [40]. The corresponding discretization system to SDDEs (17) is

$$
\begin{aligned}
x_{n+1}= & x_{n}+h x_{n}\left[r\left(1-\frac{x_{n-m_{1}}}{K}\right)-\frac{\left(1+\alpha y_{n}\right) y_{n}}{1+c\left(1+\alpha y_{n}\right) x_{n}}\right] \\
& +\sigma_{1} x_{n} \xi_{1, n}+\frac{\sigma_{1}^{2}}{2} x_{n}\left[\left(\xi_{1, n}(h)^{\frac{1}{2}}\right)^{2}-h\right], \\
y_{n+1}= & y_{n}+h y_{n}\left[\frac{\mu\left(1+\alpha y_{n}\right) x_{n-m_{2}}}{1+c\left(1+\alpha y_{n}\right) x_{n-m_{2}}}-\delta-a y_{n}\right] \\
& +\sigma_{2} y_{n} \xi_{2, n}+\frac{\sigma_{2}^{2}}{2} y_{n}\left[\left(\xi_{2, n}(h)^{\frac{1}{2}}\right)^{2}-h\right] .
\end{aligned}
$$

Here, $\xi_{1, n}$ and $\xi_{2, n}$ are mutually independent $N(0,1)$ random variables, $m_{1}, m_{2}$ are integers such that the time delays can be expressed in terms of the step-size as $\tau_{1}=m_{1} h \&$ $\tau_{2}=m_{2} h$. We provide some numerical simulations of the stochastic model (17) and its corresponding deterministic model (2). 
Taking the parameter values $\alpha=0.12, a=0.08, c=0.3, r=1, \mu=0.9, \delta=0.39, K=1$, $\sigma_{1}=\sigma_{2}=0.001$, and $\tau_{1}=\tau_{2}=0.1$, Fig. 1 shows the persistence of system (17) with initial values $(0.4,0.8)$ such that $\mathcal{R}_{0}^{s}=\frac{\mu K}{\delta(1+c)}=1.78>1$. If we increase the hunting cooperative parameter $\alpha$ as $\alpha=1.2$ keeping all other parameters the same, we can observe that the predator dominates the prey population; see Fig. 1 (right). The population densities vary around the deterministic steady state values.

Figure 2 shows the impact of small white noise on dynamics of the system. The figure displays a periodic solution of the deterministic system when $\tau_{1}=\tau_{1}^{*}=0.8 \& \tau_{2}=$
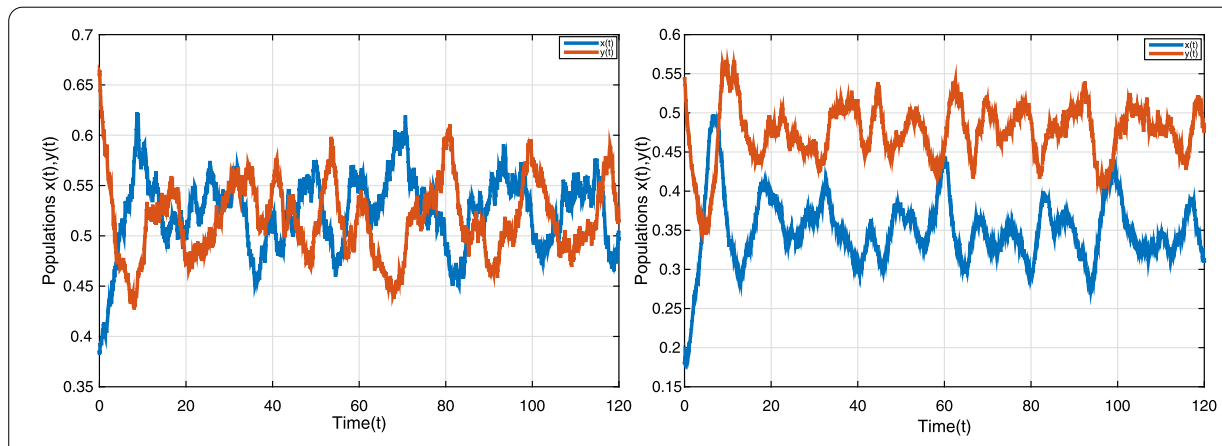

Figure 1 Numerical simulations of system (17) which display the persistence of the system, when $\mathcal{R}_{0}^{s}>1$ with $\alpha=0.12, a=0.08, c=0.3, r=1, \mu=0.9, \delta=0.39, K=1, \sigma_{1}=\sigma_{2}=0.001$, and $\tau_{1}=\tau_{2}=0.1$. However, the right banner illustrates that the predator population dominates the prey population as time goes when $\alpha$ is increased to $\alpha=1.2$
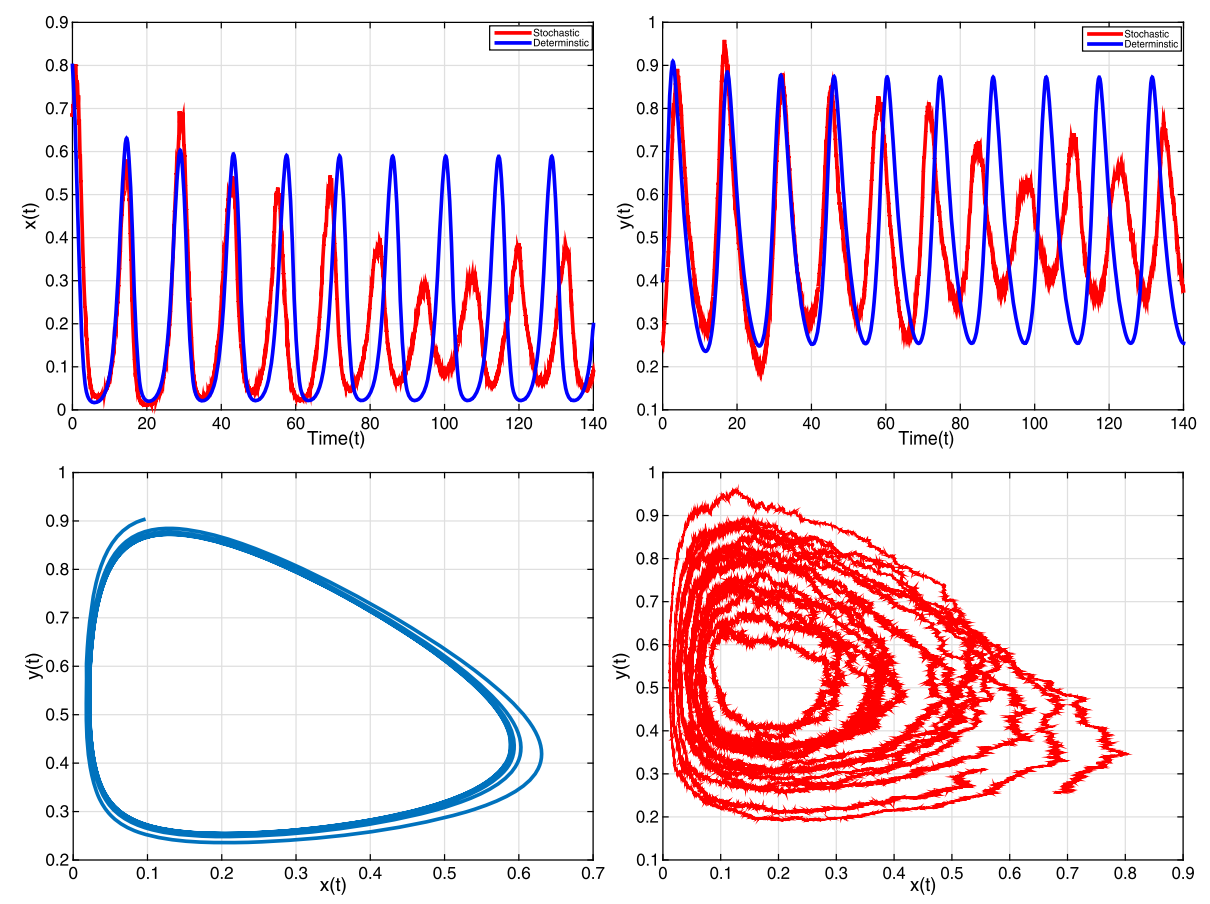

Figure 2 Numerical simulations of the solutions for system (17) and the corresponding deterministic system (2), when $\mathcal{R}_{0}^{s}>1$ with $\tau_{1}=\tau_{1}^{*}=0.8 \& \tau_{2}=0.1<\tau_{2}^{*}, a=0.08, \delta=0.19, \alpha=1.6, c=0.6, K=1, \mu=0.9, r=1$, while the intensities of Brownian motions are relatively small $\sigma_{1}=0.004 \& \sigma_{2}=0.0001$. We can observe a damped periodic solution 
$0.1<\tau_{2}^{*}, a=0.08, \delta=0.19, \alpha=1.6, c=0.6, K=1, \mu=0.9, r=1$. However, with small noises $\sigma_{1}=0.004 \& \sigma_{2}=0.0001$, where $\mathcal{R}_{0}^{s}=2.96>1$, we can observe that the periodic solution is damped in both population densities. If the intensity of white noises increases, then the predator goes to extinction, as $\mathcal{R}_{0}^{s}<1$.

Figure 3 shows that the population of prey varies around the deterministic steady state value (left), and the predator population goes to extinction at $t=70$ with the deterministic model; while with white noise at $t=20$ (right). In this simulation, we choose initial values $(x(0), y(0))=(0.8,0.4)$ and parameter values $a=0.19, \mu=0.8, \alpha=0.1, c=0.8, K=1, \delta=$ $0.59, r=1, \sigma_{1}=0.001, \sigma_{2}=0.023, \tau_{1}=\tau_{2}=0.1$. Then $\mathcal{R}_{0}^{s}=0.75<1$. According to Theorem 5 , the solution of (17) obeys $\lim \sup _{t \rightarrow \infty} \frac{\ln y(t)}{t}<0$ a.s., that is, $y(t)$ tends to zero exponentially with probability one. On the other hand, for deterministic model (2), the condition of $\mathcal{R}_{0}^{d}=\frac{\mu K}{\delta(1+c K)}=0.7<1$ is satisfied, so the boundary equilibrium point $\left(\mathcal{E}_{1}, 0\right)=(1,0)$ is a stable point.

Figure 4 shows that the environmental noise plays an important role in extinction of the predator population. When we increase the intensity of Brownian motion $B_{2}(t)$ to $\sigma_{2}=0.1, c=0.3, \mu=0.6$, and $\delta=0.4$ with other parameters as in Fig. 3, the extinction
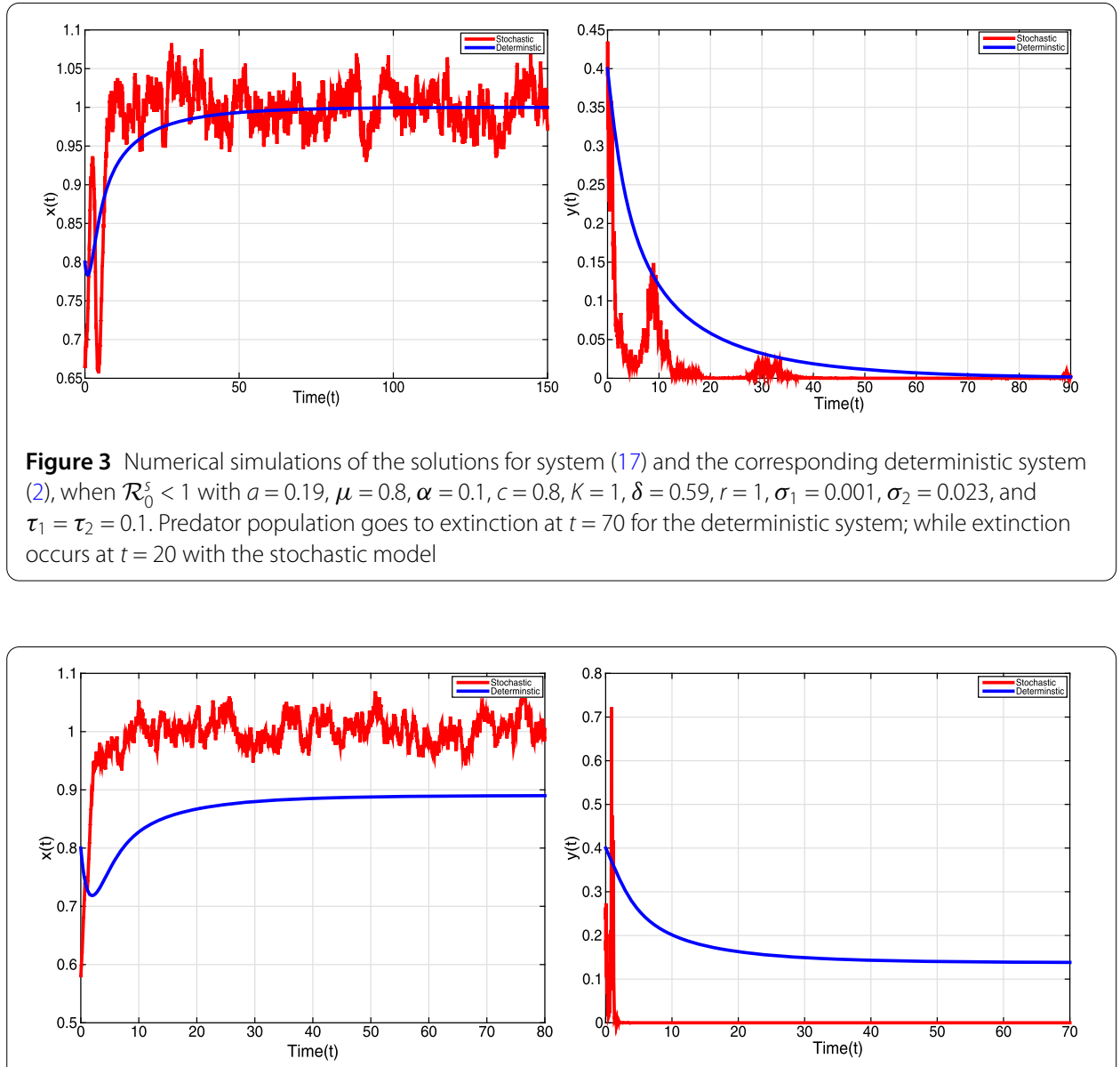

Figure 4 Numerical simulations of the solutions for system (17) and the corresponding deterministic system (2), when $\mathcal{R}_{0}^{s}<1$ with $a=0.19, \mu=0.6, \alpha=0.1, c=0.3, K=1, \delta=0.4, r=1, \sigma_{1}=0.001, \sigma_{2}=0.1$, and $\tau_{1}=\tau_{2}=0.1$. The large scale of white noises may lead to no surviving predator individuals that can reproduce and create a new generation; while the deterministic one is survival 
occurs in the predator population when $\mathcal{R}_{0}^{s}=0.92<1$. This means $y(t)$ of system (17) will go to extinction with probability one. However, with the same parameters, deterministic model (2) has an interior stable equilibrium $\mathcal{E}^{*}=(0.82,0.22)$. Therefore, the population $y(t)$ becomes extinct exponentially with probability one when white noise increases.

Remark 3 Extinction of the predator population possibly occurs when the intensity of white noise is large, such that $\mathcal{R}_{0}^{s}<1$. This would not happen in deterministic system (2) without noises (see Fig. 4). If the predator death rate is large, extinction of the predators can also occur in deterministic model (2); while with a small noise in the stochastic model, extinction of the predator population occurs faster than in the deterministic model (see Fig. 3).

\section{Discussion and conclusion}

In this paper, we studied the dynamics of SDDEs for a prey-predator system with hunting cooperation in predators. We investigated the effect of environmental fluctuations on the model. We first studied the qualitative behaviors of the deterministic model through local stability analysis of the interior equilibrium points and obtained critical values of delays, where the Hopf bifurcation occurs for the deterministic model. We then considered how environmental fluctuations affect extinction of predator and prey populations. We established the existence and uniqueness of global positive solution and the stochastically ultimate boundedness of the system. We have shown the effect of environmental noises on the persistence and possible extinction of prey and predator populations. We verified the obtained analytical results with supportive numerical simulations using Milstein's scheme. Conditions under which persistence of the system occurs have been established when $\mathcal{R}_{0}^{s}>1$; while with $\mathcal{R}_{0}^{s}<1$, extinction of the predator occurs. It can also be observed that the extinction of the predator population occurs more rapidly for stochastic system (17) when the intensity of white noise increases, see Figs. 3-4. It has also been shown numerically that the predator population dominates the prey population as cooperative hunting parameter increases (see Fig. 1).

Our main findings, theoretically and numerically, are all represented in terms of the system parameters and the intensity of randomly fluctuating driving forces. This indicates that time-delay and white noise have a considerable impact on the dynamics and presence of prey and predator populations. For future investigation, one may extend our results with other kinds of environmental noise such as color noise [41] or telephone noise [42].

\section{Appendix: Preliminaries and analysis for deterministic model}

There are three types of equilibrium points for deterministic system (2): (i) Trivial equilibrium point $\mathcal{E}_{0} \equiv(0,0)$; (ii) Axial equilibrium point $\mathcal{E}_{1} \equiv(K, 0)$; and (iii) Interior equilibrium point $\mathcal{E}^{*} \equiv\left(x^{*}, y^{*}\right)$. Here,

$$
x^{*}=\frac{\delta+a y}{(1+\alpha y)[\mu-c(\delta+a y)]} .
$$

$y^{*}$ is a positive real root of the equation

$$
\eta_{5} y^{5}+\eta_{4} y^{4}+\eta_{3} y^{3}+\eta_{2} y^{2}+\eta_{1} y^{1}+\eta_{0}=0
$$


where

$$
\begin{aligned}
& \eta_{5}=K c^{2} a^{2}, \quad \eta_{4}=2 K c^{2} a^{2} \alpha+2 \delta a K c^{2}-2 K \mu c a, \\
& \eta_{3}=K c^{2} \delta^{2}+\mu^{2} K+4 \delta a K c^{2} \alpha+K c^{2} a^{2}-2 \mu K c \delta-4 K \mu c a \alpha, \\
& \eta_{2}=2 K c^{2} \delta^{2} \alpha+2 \alpha \mu^{2} K+2 \delta a K c^{2}+r K \mu \alpha c a-4 \alpha \mu K c \delta-2 K \mu c a, \\
& \eta_{1}=K c^{2} \delta^{2}+\mu^{2} K+\mu r a+r \mu \alpha c \delta-2 \mu K \delta c-r K \mu^{2} \alpha-r K \mu a, \\
& \eta_{0}=r K \mu(c \delta-\mu) .
\end{aligned}
$$

Equation (56) must have at least one positive real root if $c \delta<\mu$. Therefore, the existence of the coexisting equilibrium $\mathcal{E}^{*}$ assumes restrictions on the parameters so that

$$
c \delta<\mu \quad \text { and } \quad y^{*} \leq \frac{\mu-c \delta}{a c} .
$$

Lemma 3 The positive solution of deterministic model (2), $(x(t), y(t))$, satisfies

$$
\lim _{t \rightarrow \infty} \sup x(t) \leq K e^{r \tau_{1}}, \quad \lim _{t \rightarrow \infty} \sup y(t) \leq \frac{\mu K e^{r \tau_{2}}-\delta}{a}
$$

for $\tau_{1}, \tau_{2}>0$ with $\mu K e^{r \tau_{2}}>\delta$.

Proof With the positive initial condition $(x(0), y(0))$, we can verify that the solution $(x(t), y(t))$ of system (2) is positive. From the first equation of system (2) we can consider

$$
\frac{d x(t)}{d t} \leq r x(t)
$$

Integrating both sides of (59) from $t-\tau_{1}$ to $t$, we have

$$
x\left(t-\tau_{1}\right) \geq x(t) e^{-r \tau_{1}} .
$$

Using (60) and from the first equation of (2), we get

$$
\frac{d x(t)}{d t} \leq x(t)\left(r-\frac{r}{K} e^{-r \tau_{1}} x(t)\right) \Rightarrow \lim _{t \rightarrow \infty} \sup x(t) \leq K e^{r \tau_{1}},
$$

i.e., for $\epsilon>0$, there exists $T_{1}>0$ such that $x(t) \leq K e^{r \tau_{1}}+\epsilon$ for all $t>T_{1}$.

Similarly, from the second equation of (2), we get

$$
\lim _{t \rightarrow \infty} \sup y(t) \leq \frac{\mu K\left(e^{r \tau_{2}}+\epsilon\right)-\delta}{a}=\xi
$$

Thus, $y(t) \leq \xi+\epsilon$ for all $t>T_{2}$, conclusion of this theorem can be achieved by letting $\epsilon \rightarrow 0$. 
Availability of data and materials

Data sharing not applicable to this paper as no datasets were generated or analyzed during the current study.

\section{Competing interests}

The authors declare that they have no competing interests.

\section{Authors' contributions}

All authors contributed equally and significantly in writing this paper. All authors read and approved the final manuscript.

\section{Publisher's Note}

Springer Nature remains neutral with regard to jurisdictional claims in published maps and institutional affiliations.

\section{Received: 29 November 2019 Accepted: 9 March 2020 Published online: 18 March 2020}

\section{References}

1. Cushing, J.M.: Structured population dynamics. In: Levin, S. (ed.) Frontiers in Mathematical Biology. Springer, Berlin (1994)

2. Gopalsamy, K.: Stability and Oscillations in Delay Differential Equations of Population Dynamics. Kluwer Academic, Dordrecht (1992)

3. Kuang, Y.: Delay Differential Equations: With Applications in Population Dynamics. Mathematics in Science and Engineering, vol. 191. Academic Press, San Diego (1993)

4. Lotka, A.: Elements of Physical Biology. Williams \& Wilkins, Baltimore (1924)

5. Volterra, V.: Variations and fluctuations in the numbers of co-existing animal species. In: Scudo, F.M., Ziegler, J.R. (eds.) The Golden Age of Theoretical Ecology: 1923-1940. Lect. Notes in Biomath., vol. 22, pp. 65-236 Springer, Berlin (1979)

6. Alves, M.T., Hilker, F.M.: Hunting cooperation and Allee effects in predators. J. Theor. Biol. 419, 13-22 (2017)

7. Owen, M.R., Lewis, M.A.: How predation can slow, stop or reverse a prey invasion. Bull. Math. Biol. 63(4), 655-684 (2001)

8. Zhang, Y., Richardson, J.S.: Unidirectional prey-predator facilitation: apparent prey enhance predators' foraging success on cryptic prey. Biol. Lett. 3(3), 348-351 (2007)

9. Saha, T., Bandyopadhyay, M.: Dynamical analysis of a delayed ratio-dependent prey-predator model within fluctuating environment. Appl. Math. Comput. 196(1), 458-478 (2008)

10. Gard, T.C.: Persistence in stochastic food web models. Bull. Math. Biol. 46(3), 357-370 (1984)

11. Rihan, F.A., Rajivganthi, C., Muthukumar, P.: Fractional stochastic differential equations with Hilfer fractional derivative: Poisson jumps and optimal control. Discrete Dyn. Nat. Soc. 2017, Article ID 5394528 (2017)

12. Ackleh, A., Allen, L., Carter, J.: Establishing a beachhead: a stochastic population model with an Allee effect applied to species invasion Theor. Popul. Biol. 71, 290-300 (2007)

13. Ji, C., Jiang, D., Li, X.: Qualitative analysis of a stochastic ratio-dependent predator-prey system. J. Comput. Appl. Math. 235, 1326-1341 (2011)

14. Hattaf, K., Mahrouf, M.: Qualitative analysis of a stochastic epidemic model with specific functional response and temporary immunity. Phys. A, Stat. Mech. Appl. 490, 591-600 (2018)

15. Yang, Q., Jiang, D.: A note on asymptotic behaviors of stochastic population model with Allee effect. Appl. Math. Model. 35, 4611-4619 (2011)

16. Liu, M., Wang, K.: Persistence, extinction and global asymptotical stability of a non-autonomous predator-prey model with random perturbation. Appl. Math. Model. 36, 5344-5353 (2012)

17. Pal, P.J., Saha, T., Sen, M., Banerjee, M.: A delayed predator-prey model with strong Allee effect in prey population growth. Nonlinear Dyn. 68(1-2), 23-42 (2012)

18. Rihan, F.A., Azamov, A.A., AlSakaji, H.J.: An inverse problem for delay differential equations: parameter estimation, nonlinearity, sensitivity. Appl. Math. Inf. Sci. 12(1), 63-74 (2018)

19. Rihan, F.A., Lakshmanan, S., Hashish, A.H., Rakkiyappan, R., Ahmed, E.: Fractional-order delayed predator-prey systems with Holling type-II functional response. Nonlinear Dyn. 80(1-2), 777-789 (2015)

20. Batzel, J.J., Tran, H.T.: Stability of the human respiratory control system I. Analysis of a two-dimensional delay state-space model. J. Math. Biol. 41(1), 45-79 (2000)

21. Bocharov, G.A., Rihan, F.A.: Numerical modelling in biosciences using delay differential equations. J. Comput. Appl. Math. 125(1-2), 183-199 (2000)

22. Kalmár-Nagy, T., Stépán, G., Moon, F.C.: Subcritical Hopf bifurcation in the delay equation model for machine tool vibrations. Nonlinear Dyn. 26(2), 121-142 (2001)

23. Hutchinson, G.E.: Circular causal systems in ecology. Ann. N.Y. Acad. Sci. 50(4), 221-246 (2948)

24. Dubey, B., Kumar, A., Maiti, A.P.: Global stability and Hopf-bifurcation of prey-predator system with two discrete delays including habitat complexity and prey refuge. Commun. Nonlinear Sci. Numer. Simul. 67, 528-554 (2019)

25. Liu, Z., Yuan, R.: Stability and bifurcation in a delayed predator-prey system with Beddington-DeAngelis functional response. J. Math. Anal. Appl. 296(2), 521-537 (2004)

26. Xu, R., Gan, Q., Ma, Z:: Stability and bifurcation analysis on a ratio-dependent predator-prey model with time delay. J. Comput. Appl. Math. 230(1), 187-203 (2009)

27. Kundu, S., Maitra, S.: Dynamical behaviour of a delayed three species predator-prey model with cooperation among the prey species. Nonlinear Dyn. 92(2), 627-643 (2018)

28. Berec, L.: Impacts of foraging facilitation among predators on predator-prey dynamics. Bull. Math. Biol. 72(1), 94-121 (2010)

29. Chunyan, J.I., Jiang, D., Liu, H., Yang, Q.: Existence, uniqueness and ergodicity of positive solution of mutualism system with stochastic perturbation. Math. Probl. Eng. 2010, Article ID 684926 (2010) 
30. Chunyan, J.., Jiang, D., Shi, N.: Analysis of a predator-prey model with modified Leslie-Gower and Holling-type II schemes with stochastic perturbation. J. Math. Anal. Appl. 359(2), 482-498 (2009)

31. Yan-Fei, J:: Moment stability for a predator-prey model with parametric dichotomous noises. Chin. Phys. B 24(6), Article ID 060502 (2015)

32. Rao, F., Castillo-Chavez, C., Kang, Y:: Dynamics of a stochastic delayed Harrison-type predation model: effects of delay and stochastic components. Math. Biosci. Eng. 15(6), 1401-1423 (2018)

33. Bandyopadhyay, M., Saha, T., Pal, R.: Deterministic and stochastic analysis of a delayed allelopathic phytoplankton model within fluctuating environment. Nonlinear Anal. Hybrid Syst. 2(3), 958-970 (2008)

34. Buckwar, E.: Introduction to the numerical analysis of stochastic delay differential equations. J. Comput. Appl. Math. 125(1-2), 297-307 (2000)

35. Mao, X:: Stochastic Differential Equations and Applications. Elsevier, Amsterdam (2007)

36. Heinrich, W.: Arnold, L., Stochastic Differential Equations, Theory and Applications, New York. John Wiley \& Sons. 1974 XVI, 228 S., £ 9.50 (engl. Übersetzung des deutschen Originals, R. Oldenbourg 1973). Z. Angew. Math. Mech. 57, 271 (1977)

37. Mao, X.: Stochastic Differential Equations and Their Applications. Horwood, Chichester (1997)

38. Liptser, R.S.: A strong law of large numbers for local martingales. Stochastics 3(1-4), 217-228 (1980)

39. Ji, C., Jiang, D.: Threshold behaviour of a stochastic SIR model. Appl. Math. Model. 38(21-22), 5067-5079 (2014)

40. Kloeden, P.E., Shardlow, T.: The Milstein scheme for stochastic delay differential equations without using anticipative calculus. Stoch. Anal. Appl. 30(2), 181-202 (2012)

41. Lawton, J.H.: More time means more variation. Nature 334(6183), 563 (1988)

42. Li, D., Liu, S., Cui, J.: Threshold dynamics and ergodicity of an SIRS epidemic model with Markovian switching. J. Differ. Equ. 263(12), 8873-8915 (2017)

\section{Submit your manuscript to a SpringerOpen ${ }^{\circ}$ journal and benefit from:}

- Convenient online submission

- Rigorous peer review

- Open access: articles freely available online

- High visibility within the field

- Retaining the copyright to your article

Submit your next manuscript at $\boldsymbol{\Delta}$ springeropen.com 